\title{
A Liquid-Lithium Target project for production of high-intensity quasi-stellar neutrons
}

\author{
Gitai Feinberg, Alexander Arenshtam, Dan Berkovits, Yosef Eisen, Shlomi \\ Halfon, Dany Kijel, Ami Nagler, Asher Shor and Ido Silverman \\ Soreq Nuclear Research Center, Yavne, Israel \\ E-mail: gitai.feinberg@gmail.com
}

\section{Michael Paul and Moshe Friedman}

Racah Institute of Physics, The Hebrew University, Jerusalem, Israel

\begin{abstract}
A windowless Liquid-Lithium Target (LiLiT) is under construction and development at Soreq NRC (Israel). The target is designed to be bombarded by a 2-4 mA proton beam $\left(\mathrm{E}_{\mathrm{p}}=1.8-2.5\right.$ $\mathrm{MeV}$ ) from the high-intensity Soreq Applied Research Accelerator Facility (SARAF), a superconducting linear accelerator for light ions. The liquid-lithium forced flow at a velocity of $\sim 20 \mathrm{~m} / \mathrm{s}$ and a thickness of $\sim 1.8 \mathrm{~mm}$ serves both as a power dump (10 kW) for the proton beam and as a neutron-producing target via the ${ }^{7} \mathrm{Li}(p, n)^{7} \mathrm{Be}$ reaction. As known from the work of the Forschungszentrum Karlsruhe group, the energy distribution of neutrons emitted for a proton energy $E_{p}=1.912 \mathrm{MeV}, \sim 30 \mathrm{keV}$ above the reaction threshold, and a thick Li target is very similar to that of a Maxwell-Boltzmann flux at a thermal energy of $\sim 25 \mathrm{keV}$, well suited for activation measurements relevant to $s$-process nucleosynthesis. The neutron intensity expected under these conditions from the combination of the SARAF proton beam and the LiLiT thermal properties is of $\sim 2 \times 10^{10} \mathrm{~s}^{-1} \mathrm{~mA}^{-1}$, and is larger by more than one order of magnitude than currently available. The LiLiT setup is built as a loop circulating liquid lithium at a temperature of $\sim 200^{\circ} \mathrm{C}$ and producing a jet (acting as the target) onto a thin concave supporting wall, driven by a rotating magnet inductive electromagnetic pump. The liquid lithium is collected in a reservoir housing a heat exchanger with a mineral-oil closed loop. Circulation and thermal tests of the loop are presently in progress in an offline dedicated electron-gun laboratory and online installation at the SARAF accelerator is planned for end 2010. Characterization of the SARAF proton beam (beam energy, energy width and transverse profile) and of the neutron spectrum obtained under these conditions are studied in parallel using a solid-lithium (lithium fluoride) target at low beam intensities. The SARAF-LiLiT system will be used to measure stellar neutron capture cross sections for stable or radioactive targets demanding high neutron fluxes. Present status and plans are discussed.
\end{abstract}

11th Symposium on Nuclei in the Cosmos - NIC XI

Heidelberg, Germany

July 19-23 2010 


\section{Introduction}

A windowless Liquid-Lithium Target (LiLiT) is in construction and development at Soreq NRC (Israel). The target is designed to be bombarded by a 2-4 $\mathrm{mA}$ proton beam $\left(\mathrm{E}_{\mathrm{p}}=1.8-2.5\right.$ $\mathrm{MeV}$ ) from the high-intensity accelerator in the Soreq Applied Research Accelerator Facility (SARAF) $)^{(1)}$, a superconducting linear accelerator for light ions. The liquid-lithium forced flow at a velocity of $\sim 20 \mathrm{~m} / \mathrm{s}$ and a thickness of $\sim 1.8 \mathrm{~mm}$ serves both as the neutron-producing target via the ${ }^{7} \mathrm{Li}(p, n)^{7} \mathrm{Be}$ reaction and as power dump $(10 \mathrm{~kW})$ for the proton beam. The SARAFLiLiT system will be used to measure stellar neutron capture cross sections for stable or radioactive targets demanding high neutron fluxes.

\section{SARAF phase I}

The SARAF is based on a continuous wave $(\mathrm{CW})$, proton/deuteron RF superconducting linear accelerator with currents up to $2 \mathrm{~mA}$, upgradeable to $4 \mathrm{~mA}^{(1)}$. Phase I of SARAF presently in final commissioning will produce proton and deuteron beams at full intensity up to 3-5 MeV. A temporary experimental beam line is being built and a high-intensity proton beam on target is expected in 2010. Phase II with a full energy of $40 \mathrm{MeV}$ is planned for 2015.
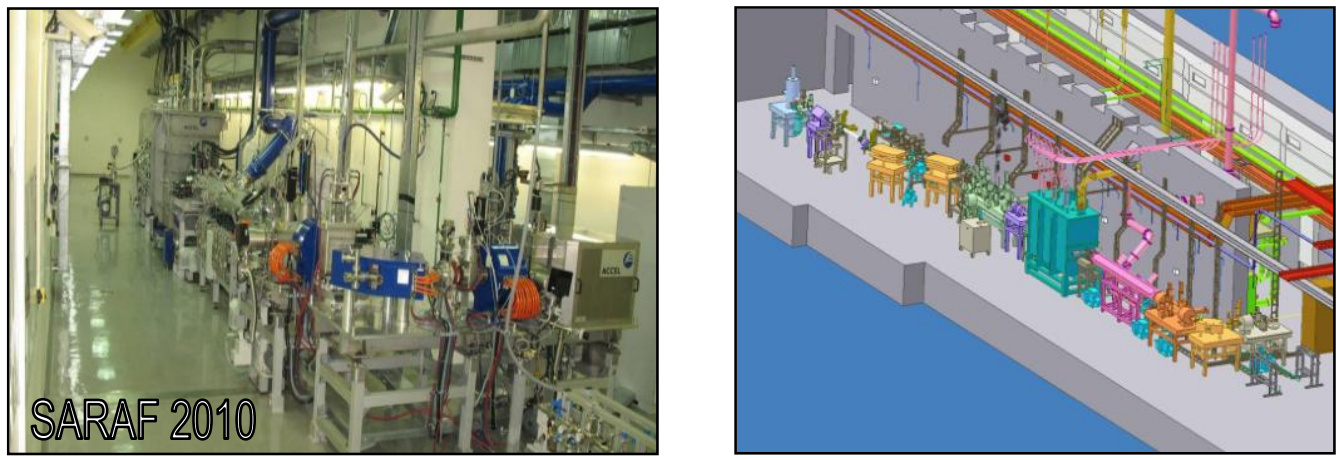

Figure 1: SARAF phase I.

\section{LiLiT - Liquid-Lithium Target}

The LiLiT setup ${ }^{(2)}$ is built as a loop circulating liquid lithium at a temperature of $\sim 200^{\circ} \mathrm{C}$ and producing a jet (acting as the target) onto a thin concave supporting wall, driven by a rotating magnet inductive electromagnetic pump. The liquid lithium is collected in a reservoir housing a heat exchanger with a mineral-oil closed loop.
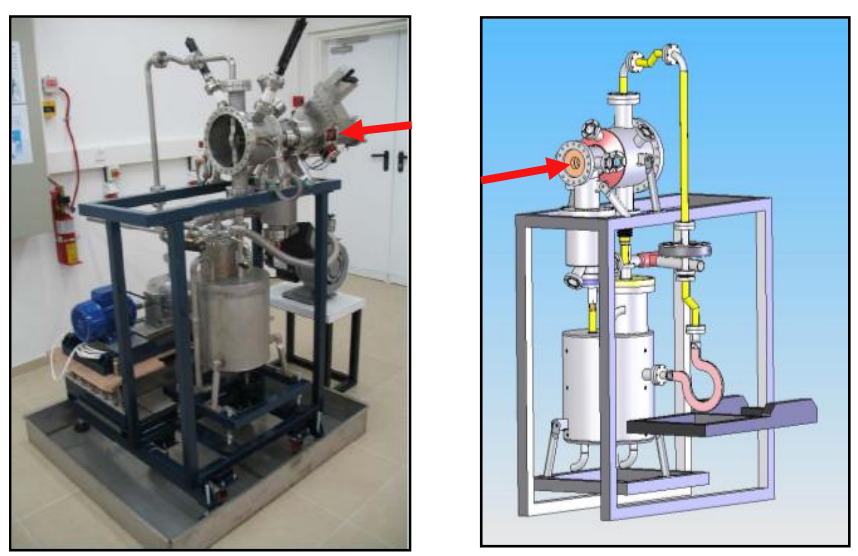

Figure 2: The LiLiT loop. The red arrow represents the direction of the proton beam. 

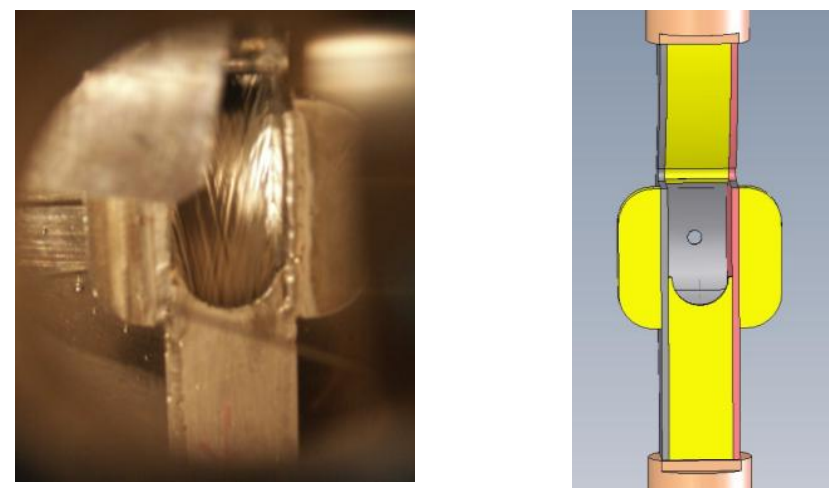

Figure 3: Left - lithium flowing in the nozzle. Right - 3D schematic drawing of the nozzle.

\section{Neutron spectrum calculations}

Calculations were performed for the neutron spectrum produced by the ${ }^{7} \mathrm{Li}(p, n)^{7} \mathrm{Be}$ reaction. When the proton energy is $1912 \mathrm{keV}$, neutrons are emitted in a cone of $\pm 60^{\circ}$ angular opening and the calculated neutron energies, using the ${ }^{7} \mathrm{Li}(p, n)^{7} \mathrm{Be}$ energy dependent cross section and integration over all incident energies down to threshold in a thick Li target and over all neutron angles, result in a spectrum very close to a $E e^{-E / k T}$ dependency with an effective value $k T=26 \mathrm{keV}^{(3)}$. The energy distribution is close to that of a flux of Maxwellian neutrons at this temperature. In these conditions the neutron spectrum has a cutoff at $107 \mathrm{keV}$ and the neutron yield is $\sim 2.5 \times 10^{10}$ neutrons $/ \mathrm{mA} / \mathrm{s}$. The neutron spectrum calculated for the SARAF proton beam with an energy spread $(1 \sigma=8 \mathrm{keV}$ in the present simulation) extends to higher energies and fits better the high-energy tail of the Maxwellian flux (Fig. 4 right).
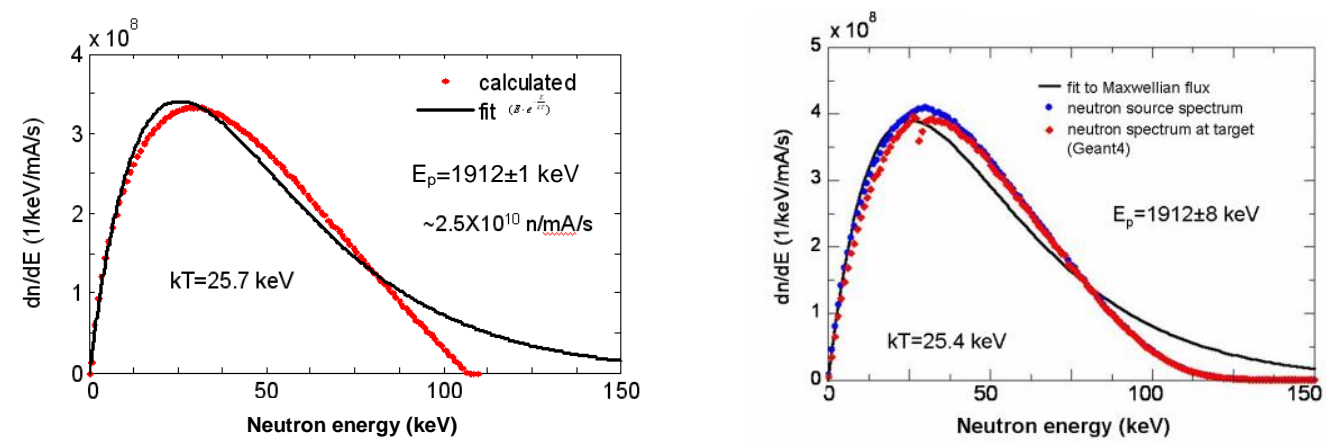

Figure 4: (left) Calculated neutron spectrum for a proton beam with low-energy spread and fit for a Maxwellian flux; (right) simulation including the effects of a 8-keV energy spread in the proton beam and of surrounding materials. The simulation was done with the code GEANT4 ${ }^{(4)}$.

\section{MACS measurements}

Maxwellian Averaged capture Cross Section (MACS) measurements are planned. As schematically illustrated in fig. 5 , the samples will be placed close to the LiLiT vacuum window. We will minimize the material in the area of the

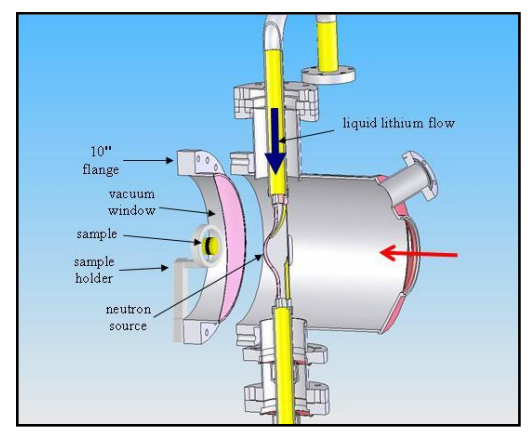

Figure 5: The experimental setup. 
sample to reduce the effect of scattered neutrons on the activation, although simulations show a minimal effect only (fig 4 , right).

\section{Thermal behavior}

Simulations were performed to estimate the temperature rise in the lithium jet, for a jet velocity of $20 \mathrm{~m} / \mathrm{s}$ irradiated by a $2 \mathrm{MeV}, 2 \mathrm{~mA}$ proton beam. From a baseline temperature of $200^{\circ} \mathrm{C}$, the temperature rises up to a maximum of $280^{\circ} \mathrm{C}$ on the lithium surface $5 \mathrm{~mm}$ downstream the center of the proton beam and the overall temperature rise of the whole lithium volume is $4^{0}$. Depending on the loop parameters, stable operation may be achieved for

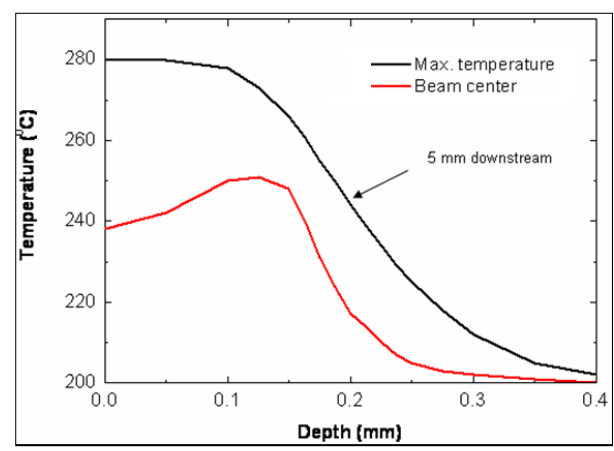
lower jet velocities.

Figure 6: Temperature profile in the lithium jet.

\section{Beam dynamics simulations}

Preliminary beam dynamics simulations for the SARAF phase I accelerator and beam line were performed using the GPT simulation code ${ }^{(5)}$. The simulation started at the RFQ exit (see ref. 1) based on a calculated proton distribution. The RMS radius of the proton beam at target position is $3.1 \mathrm{~mm}$ and the energy spread (1 STD) is $7 \mathrm{keV}$. Energy is mainly gained using cavities 3 and 4, cavities 1 and 6 operate as bunchers while cavities 2 and 5 are turned off for a $1.912 \mathrm{MeV}$ final energy and low energy spread. The beam line design is not finalized but based on the simulation the required beam parameters can be achieved with reasonably low energy proton losses.
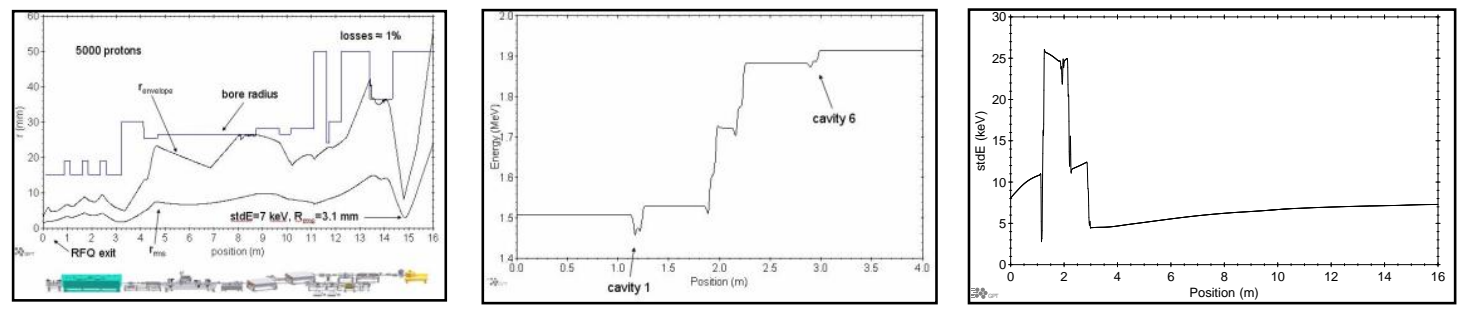

Figure 7: Beam dynamics calculations. Left to right - transverse, energy and energy spread behavior along the linac and beam line.

\section{References}

[1] I. Mardor et al., in proceedings of SRF2009, Berlin, Germany, MOODAU04, 74 (2009).

[2] G. Feinberg et al., Nucl. Phys. A 827, 590c (2009).

[3] W. Ratynski and F. Kaeppeler, Phys. Rev. C 37, 595 (1988).

[4] S. Agostinelli et al., Nucl. Instr. Meth. A, 506 (3), 250 (2003).

[5] GPT (General Particle Tracer), release 2.8, Pulsar Physics Ltd, (2007). 